\title{
Control of evaporating complex fluids through electrowetting
}

\author{
D. Mampallil, H. B. Eral, D. van den Ende and F. Mugele*
}

Received 12th May 2012, Accepted 3rd July 2012

DOI: $10.1039 / \mathrm{c} 2 \mathrm{sm} 26103 \mathrm{k}$

Evaporating drops of complex fluids such as colloidal suspensions and macromolecular solutions typically leave behind ring-shaped solid residues commonly known as coffee stains. Electrowettingdriven microfluidic flows allow for controlling this process. We present coffee stain suppression for colloidal suspensions of variable concentration and particle size and we demonstrate an improved sample preparation method for MALDI mass spectrometry.

Coffee stains are a ubiquitous phenomenon accompanying the evaporation of complex fluids including colloidal suspensions and solutions of macromolecules such as polymers and biomolecules. The occurrence of coffee stains is caused by a combination of solvent evaporation and contact line pinning: ${ }^{1}$ whenever the contact line of an evaporating drop is prevented from receding, a convective flux is generated from the center towards the edge of the drop in order to sustain the solvent evaporation. This flux carries solute from the bulk of the drop towards the contact line leading to a local increase of the concentration and eventually to precipitation of the solute. In many applications including inkjet printing, spotting of biofluids, and coating technology the resulting highly inhomogeneous solute deposition is undesired., ${ }^{2,3}$ The request for more homogeneous deposition prompted various attempts to suppress coffee stain formation. To achieve this goal it is necessary to prevent contact line pinning and/or to prevent the accumulation of solute near the contact line. The former can be achieved by using homogeneous hydrophobic surfaces that display little intrinsic contact angle hysteresis. At the same time, the large contact angles reduce the otherwise diverging evaporation rate along the contact line. For ellipsoidal particles that are adsorbed strongly onto the air-water interface, interfacial gelation and geometric blockage can also prevent the solute particles from reaching the contact line and thereby reduce coffee stain formation. ${ }^{4}$ Alternatively, solute accumulation at the contact line can be suppressed by generating flow patterns within the drop that counteract the evaporation driven flux. The most widely used strategy to achieve this makes use of Marangoni flows, ${ }^{5-7}$ which can be driven either by temperature gradients that are caused by the evaporation process itself or by surfactants added to the system. Recently, we demonstrated that electrowetting (EW) can also be used to suppress coffee stain formation. ${ }^{8}$ EW-based coffee stain suppression was shown to be

University of Twente, MESA+ Institute for Nanotechnology, Physics of Complex Fluids, P.O. Box 217, 7500 AE Enschede, The Netherlands. E-mail:f.mugele@utwente.nl; Fax: +31 53489 1097; Tel: +31 534893094 effective both for suspensions of colloidal particles and for DNA molecules at low volume fractions and concentrations, respectively.

In this communication, we extend our first study towards a more complete discussion of the physical effects underlying the EW-based coffee stain suppression and explore its efficiency for colloidal suspensions of variable volume fraction and size. Moreover, we demonstrate that the deposition of all the solute in a single spot rather than a widely spread heterogeneous coffee stain can improve the efficiency of MALDI (matrix-assisted laser desorption ionization) mass spectrometry by more than one order of magnitude.

Fig. 1 illustrates the basic principle of the experiment. Sessile drops of colloidal suspension with an initial volume of $0.5 \mu \mathrm{L}$ are deposited onto a solid surface and left to evaporate. During the evaporation process, the contact line of the drop can be either pinned (a) or freely moving (b). In the former case, the radius of the drop-substrate
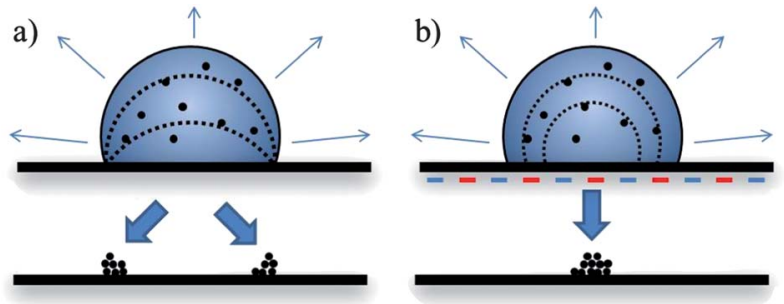

c)
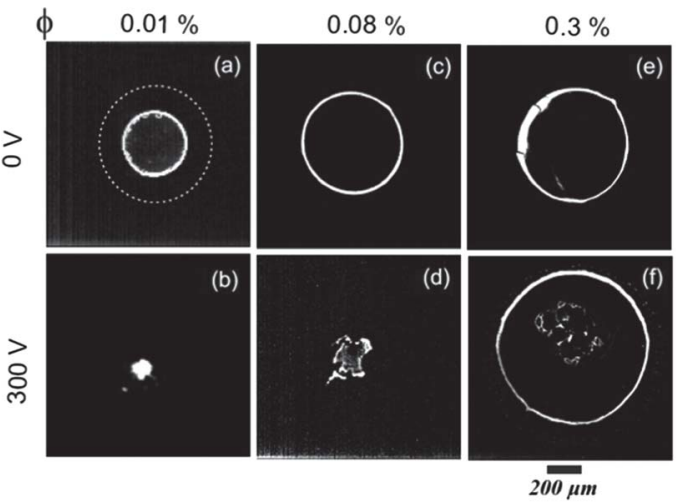

Fig. 1 Schematic of coffee stain formation for an evaporating drop with pinned contact lines (a) and with mobile contact lines (b) leading to solute concentration in a single spot. Contact line mobilization is achieved by applying an AC voltage between the interdigitated electrodes (short red and blue lines) in (b). (c) Snapshots of residues of PS particles (diam. $100 \mathrm{~nm}$ ) after evaporation without (top row) and with electrowetting at $300 \mathrm{~V}$ and $1 \mathrm{kHz}$ (bottom row) for three different volume fractions. 
interface remains constant while the contact angle gradually decreases. As explained by Deegan et al. ${ }^{1}$ this results in an internal flow that carries the solute towards the contact line where it eventually precipitates to form the characteristic ring-shaped solid residue. For millimetre-sized drops, the typical velocity of this flow is initially of the order of $10 \mu \mathrm{m} \mathrm{s}^{-1}$ and diverges as the contact angle vanishes towards the final state of the evaporation process. ${ }^{1,11}$ In contrast, for perfectly smooth solid surfaces with freely moving contact lines, the contact angle remains constant and the drop-substrate area gradually shrinks during the evaporation process (Fig. 1b). In this case, the residual solute is concentrated in a single spot. In practice, solid surfaces with low contact angles and hence a vanishing receding contact angle typically follow the first scenario whereas poorly wetting substrates with receding contact angles $>90^{\circ}$ follow the second scenario. Surfaces with intermediate contact angles tend to follow a mixed scenario, where the contact line initially recedes at the receding contact angle and at some point gets pinned e.g. if the increasing concentration of solute particles leads to the precipitation of clusters that stick to the solid surface. The top row of Fig. 1c shows the final states of a series of evaporating drops following this mixed scenario with a variable initial concentration of carboxylated polystyrene (PS) particles on a thin polymer film (SU8; thickness $d=5$ $\mu \mathrm{m})$ with an advancing and receding contact angle for pure water of $\theta_{\mathrm{a}}=85 \pm 3^{\circ}$ and $\theta_{\mathrm{r}}=60 \pm 3^{\circ}$, respectively. For low solute concentrations, the contact line initially recedes and gets pinned at some later state. For higher concentrations, pinning occurs immediately. Under these conditions, it is possible to suppress the coffee stain formation by exciting the drop during the evaporation process with electrowetting, as shown in the bottom row of Fig. 1c. Provided that the initial concentration is not too high, excitation of the drop with alternating (AC) voltage in a broad frequency range (a few $\mathrm{Hz}$ to several $\mathrm{kHz}$ ) can prevent the contact line from pinning to surface defects. ${ }^{8}$ This eventually leads to the desired deposition of solute particles in a single small spot. Beyond a certain threshold concentration, however, this process is no longer effective and a coffee stain is formed (Fig. 1c, bottom right). The radius of this stain is even somewhat larger than in the absence of applied voltage due to the initial spreading of the drop upon applying the EW voltage. Similar data were obtained for aqueous solutions of DNA. ${ }^{8}$

The observed suppression of the coffee stain effect is caused by a combination of two effects, as illustrated in Fig. 2. In the absence of $\mathrm{EW}$, surface tensions $\sigma_{\mathrm{i}}$ of the solid-liquid (i=sl), solid-vapor (sv), and liquid-vapor (lv) interfaces are the primary forces acting on the contact line. They are complemented by random pinning forces $f_{\mathrm{p}}$ caused by the heterogeneity of the substrate, which are the source of contact angle hysteresis. In this simple one-dimensional picture, the contact line can only advance or recede if the contact angle $\theta$ deviates sufficiently from Young's angle $\theta_{\mathrm{Y}}$ to overcome the pinning forces. In the presence of EW, the electrostatic force (per unit length) $f_{\mathrm{el}}=$ $c U^{2} / 2$ additionally pulls on the contact line in the outward direction. Here, $U$ is the applied voltage and $c=\varepsilon \varepsilon_{0} / d$ is the capacitance per unit area between the drop and the electrode (dielectric constant $\varepsilon=3$ for SU8). This additional force causes the reduction of the equilibrium contact angle in EW following

$$
\cos \theta=\cos \theta_{\mathrm{Y}}+\eta
$$

Here, $\eta=f_{\mathrm{el}} / \sigma_{\mathrm{lv}}$ is the dimensionless electrostatic force, also known as electrowetting number. ${ }^{12}$ Since $f_{\mathrm{el}}$ simply adds to the force balance, it
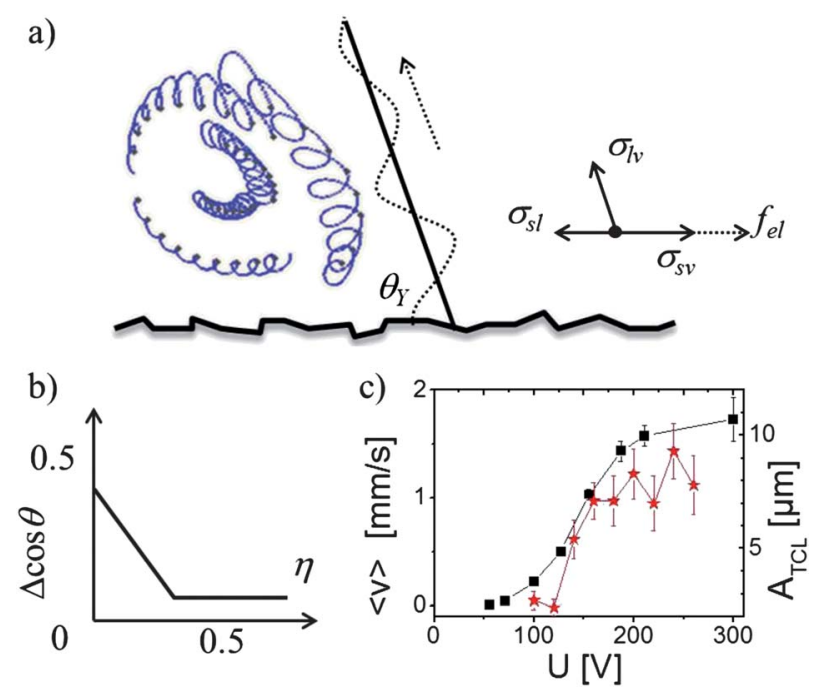

Fig. 2 (a) Schematic of oscillatory contact line motion and the accompanying propagating capillary wave (dotted line) at the drop surface along with resulting trajectories of colloidal tracer particles (numerical trajectories adapted from ref. 9). Right: force balance at the contact line including electrical force $f_{\mathrm{el}}$. (b) Contact angle hysteresis vs. electrowetting number expressed as $\Delta \cos \theta=\cos \theta_{\mathrm{r}}-\cos \theta_{\mathrm{a}}$. (after ref. 10). (c) Average flow velocity (black squares) and amplitude of contact line (red stars) vs. applied voltage for a drop on the SU8 surface.

reduces the advancing and receding contact angles by the same amount in the case of DC voltage. For an AC voltage, $f_{\mathrm{el}}$ can be decomposed into a time-averaged root-mean-square component and a time-dependent component oscillating at twice the applied electrical frequency $\omega: f_{\mathrm{el}}=\sigma_{\mathrm{lv}} \eta(1+\cos 2 \omega t)$. For excitation frequencies in the $\mathrm{kHz}$ range, i.e. well above the lowest eigen frequencies of the drops, the former part gives rise to a reduction of the time-averaged contact angle following eqn (1) while the latter remains uncompensated by the bulk drop and is available to depin the contact line from local defects. This reduces the macroscopically measurable contact angle hysteresis $^{10}$ and allows mobilization of pinned contact lines and drops ${ }^{13}$ as illustrated in Fig. 2b. If continuously applied during the evaporation process, this prevents pinning of contact lines and hence prevents colloidal particles from sticking to the substrate. This is the first mechanism to suppress coffee stain formation. (A similar effect can also be achieved by low frequency modulations of the applied voltage at large amplitudes. ${ }^{8}$ ) A second effect counteracting coffee stain formation is caused by the dynamic response of the drop to the electrical AC excitation. As mentioned above, the excitation frequencies are too high for the entire drop to follow. Yet, locally, the liquid can respond and the contact line is in fact moving back and forth at the oscillation frequency of $f_{\mathrm{e}}$, i.e. at $2 \omega$. This local response leads to the generation of capillary waves that travel from the contact line upwards towards the apex of the drop as sketched in Fig. 2a. These travelling waves induce a net flow of fluid upward along the drop surface as in the so-called Stokes drag mechanism studied earlier in the context of mixing oscillating aqueous drops in ambient oil. ${ }^{14,15}$ In that system, high speed tracking of passive colloidal tracer particles $^{15}$ as well as numerical calculations ${ }^{9}$ show a net drift flow superimposed onto a periodic oscillatory motion, which leads to a spiralling motion of the particles moving along with the travelling capillary wave. A typical trajectory of a particle taken from Oh et al. ${ }^{9}$ is shown in Fig. 2a. For evaporating drops in air the flow patterns are 
slightly more complex and frequently display symmetry breaking in the azimuthal plane in addition to the polar convection role sketched in Fig. 2a. Rather than axisymmetric flow fields, we typically observe a two- or four-fold symmetry in the drop-substrate plane similar to experiments with one or two intentionally added pinning sites along the contact line. ${ }^{16}$ We attribute these patterns to contact angle hysteresis, which is strongly enhanced when compared to the idealized case of ambient oil. (Depending on the specific conditions, mechanisms other than Stokes drift can also give rise to strong internal flow patterns. ${ }^{17-19}$ ) Fig. $2 \mathrm{c}$ shows the correlation between the average flow velocity and the motion of the contact line as a function of the applied voltage for the aqueous suspensions of PS particles present on SU8 substrates. For voltages beyond $200 \mathrm{~V}$ both the flow speed and the amplitude of the contact line (measured by high speed video microscopy) become saturated due to the onset of contact angle saturation in the present system. The strength of these convective flows exceeds that of the typical evaporation driven flows by more than one order of magnitude. As a consequence, they completely prevent any accumulation of solute at the contact line. Together the direct depinning under the influence of $f_{\mathrm{el}}$ and the redistribution of solute due to the secondary flow cause the suppression of coffee stains shown in Fig. 1.

The formation of coffee stains is known to depend on a variety of parameters including the concentration, size, and interactions of the solute particles and the contact angle and contact angle hysteresis of the substrate. To evaluate the efficiency of the EW mechanism for coffee stain suppression we systematically varied the size and concentration of the solute particles for the fixed system of carboxylated PS particles and SU8 surfaces. PS particles with diameters between $20 \mathrm{~nm}$ and $3 \mu \mathrm{m}$ were suspended in $10 \mathrm{mM} \mathrm{LiCl}$ solutions in demineralized water at volume fractions ranging from 0.0007 to $0.6 \%$. The $\mathrm{pH}$ was not controlled and assumed a typical value of around 6 . For each type of suspension, we evaporate drops at various voltages and inspect the size of the final residues, as in Fig. 1b. Coffee stain formation is considered to be suppressed if the diameter of the final residue is less than $40 \%$ of the initial drop size at zero voltage. For each condition (particle size and concentration), we determine the critical voltage $V_{\mathrm{c}}$ above which coffee stains are suppressed for a fixed excitation frequency of $1 \mathrm{kHz}$. We find that $V_{\mathrm{c}}$ increases both with increasing concentration and with decreasing particle size. Fig. 3

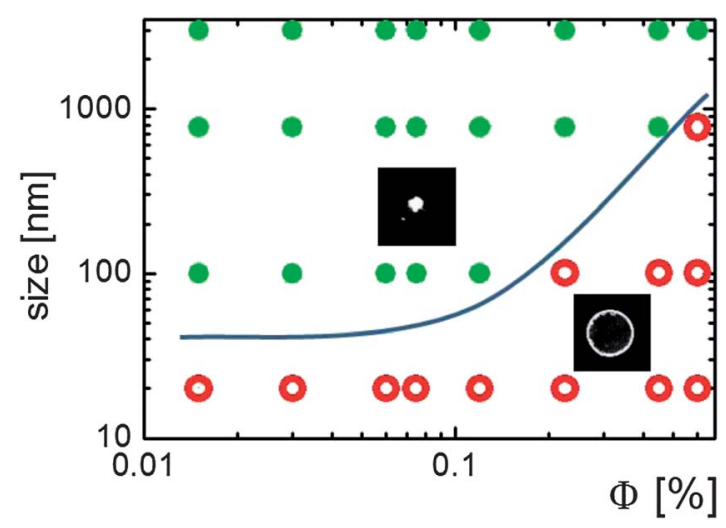

Fig. 3 Coffee stain suppression for carboxylated PS particles as a function of volume fraction and particle size. Green: successful suppression; red: unavoidable coffee stain formation. The solid line visually separates the two regimes. (EW voltage: $300 \mathrm{~V}$ at $1 \mathrm{kHz}$.) summarizes the regimes of concentrations and particle diameters for which coffee stains can be suppressed. For particle sizes of $20 \mathrm{~nm}$ it is not possible to suppress coffee stain formation even at the lowest concentrations and the highest voltage $(300 \mathrm{~V})$ available in the present experiments. This suggests that the colloidal particles blocking the contact line motion stick rather strongly to the substrate surface. For larger particles, it is apparently easier to keep the contact line mobile during the evaporation process and hence to suppress coffee stain formation. This suggests that the adhesive forces increase more weakly with increasing particle size than the hydrodynamic drag forces. We suspect that the particle-substrate interaction plays an important role for the onset of contact line pinning and thus for the overall limits of the EW-controlled coffee stain suppression with respect to particle size and concentration. A more detailed modelling of this process is beyond the purpose of the present communication. Clearly such models, which may depend very much on the specific system, should contain the relevant colloidal interactions including electrostatics, van der Waals forces, and the more complex hydrophobic interactions.

As a second example, we demonstrate that EW-controlled coffee stain suppression can help to improve bioanalytical MALDI MS (matrix-assisted laser desorption ionization mass spectrometry). Briefly, coffee stain formation is a problem in MALDI MS because drops of the analyte solution are usually spotted together with an organic salt, the matrix, onto a solid carrier surface. The drops are left to evaporate. Subsequently, the dried residues are irradiated by a UV laser pulse. The laser light is absorbed by the matrix. The analyte is vaporized along with the matrix, becomes charged and is subsequently analysed in the mass spectrometer. Coffee stain formation during the evaporation process frequently leads to a very inhomogeneous deposition of both the matrix and the analyte distributed of an area that is very wide compared to the typical diameter of the laser spot. As a consequence, the laser has to be scanned across the sample surface and spectra from different locations with widely varying intensities need to be averaged. Alternatively, one has to search manually for "hot spots" on the sample providing strong signals. If coffee stain formation is suppressed using EW, the sample is

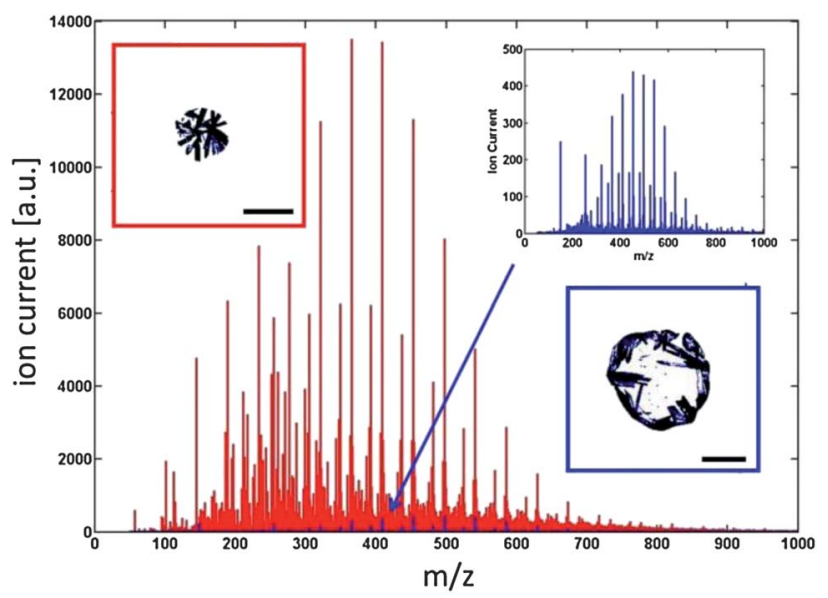

Fig. 4 MALDI spectrum of a typical biological reference sample of PEG solution in DHB buffer (main graph). Red: with EW; blue without EW. Insets top left and bottom right show solid residues (matrix crystals with analyte) with and without EW, respectively (scale bars: $200 \mu \mathrm{m}$ ). Top right: $10 \times$ magnified MALDI spectrum with EW. See text for details. 
distributed more homogeneously in a smaller spot leading to a stronger MALDI signal. In Fig. 4, we illustrate the process using a standard test sample. We deposit drops of PEG (polyethylene glycol; concentration: $0.01 \mu \mathrm{M}$ ) with an average mass of $400 \mathrm{amu}$ dissolved in an aqueous solution of the matrix DHB (dihydroxybenzoic acid; conc.: $1.8 \mathrm{mg} \mathrm{mL}^{-1}$ ). In the absence of EW, crystallization of the matrix sets in rather early in the evaporation phase, leading to large crystallites that grow preferentially from the contact line toward the center of the drop (see snapshot with blue border in Fig. 4). Integrating the signal from the mass spectrometer over all laser shots, we obtain the MALDI spectrum shown in blue in the main panel and magnified on the top right. If we apply EW during the evaporation process, crystallization of the matrix sets in at a later stage leading to a smaller and more concentrated deposition of the matrix and analyte on the surface (snapshot with red border). The resulting integrated MALDI spectrum is approximately $30 \times$ stronger than one obtained without EW. While a detailed analysis of the performance of the method for a wider spectrum of samples and matrix materials will be reported elsewhere, these preliminary results already indicate that EW provides a promising route to improved bioanalytic mass spectrometry. We denote this process as eMALDI, ${ }^{20}$ which may be combined with other strategies of EW-based MALDI sample pretreatment. $^{21}$

\section{Conclusions}

We demonstrate that electrowetting is a versatile tool to control and suppress the formation of coffee stains from evaporating drops of complex fluids. For colloidal suspensions of polystyrene particles, the process is limited to volume fractions below $1 \%$ and to particle diameters of $100 \mathrm{~nm}$ and larger. For evaporating drops of aqueous polyethylene glycol solutions co-evaporated with an organic salt, we find a promising enhancement of MALDI mass spectrometry signals upon suppressing coffee stain formation with electrowetting. In both cases more detailed experiments are needed to pinpoint the physical effects governing the efficiency of the process, such as the initial stages of contact line pinning and the nucleation of matrix crystals. Note that EW may also affect other crystallization processes such as protein crystallization.

\section{Acknowledgements}

We thank H. Gardeniers for providing access to the MALDI machine. Funding by the Dutch National Science Foundation NWO and by MicroNed, the Microtechnologies Program of The Netherlands is acknowledged.

\section{Notes and references}

1 R. D. Deegan, O. Bakajin, T. F. Dupont, G. Huber, S. R. Nagel and T. A. Witten, Nature, 1997, 389, 827.

2 E. Tekin, P. J. Smith and U. S. Schubert, Soft Matter, 2008, 4, 703 .

3 R. G. Larson, Angew. Chem., Int. Ed., 2012, 51, 2546.

4 P. J. Yunker, T. Still, M. A. Lohr and A. G. Yodh, Nature, 2011, 476, 308.

5 H. Hu and R. G. Larson, Langmuir, 2005, 21, 3972.

6 W. D. Ristenpart, P. G. Kim, C. Domingues, J. Wan and H. A. Stone, Phys. Rev. Lett., 2007, 99, 234502.

7 T. Still, P. J. Yunker and A. G. Yodh, Langmuir, 2012, 28, 4984.

8 H. B. Eral, D. M. Augustine, M. H. G. Duits and F. Mugele, Soft Matter, 2011, 7, 4954.

9 J. M. Oh, D. Legendre and F. Mugele, EPL, 2012, 98, 34003.

$10 \mathrm{~F}$. Li and F. Mugele, Appl. Phys. Lett., 2008, 92, 244108.

11 A. G. Marin, H. Gelderblom, D. Lohse and J. H. Snoeijer, Phys. Rev. Lett., 2011, 107, 085502.

12 F. Mugele and J. C. Baret, J. Phys.: Condens. Matter, 2005, 17, R705R774.

13 D. T. Mannetje, C. U. Murade, D. van den Ende and F. Mugele, Appl. Phys. Lett., 2011, 98, 014102.

14 F. Mugele, J. C. Baret and D. Steinhauser, Appl. Phys. Lett., 2006, 88, 204106.

15 F. Mugele, A. Staicu, R. Bakker and D. van den Ende, Lab Chip, 2011, 11, 2011.

16 D. Mampallil, D. van den Ende and F. Mugele, Appl. Phys. Lett., 2011, 99, 154102 .

17 S. H. Ko, H. Lee and K. H. Kang, Langmuir, 2008, 24, 1094.

18 S. H. Ko, S. J. Lee and K. H. Kang, Appl. Phys. Lett., 2009, 94, 194102.

19 P. Garcia-Sanchez, A. Ramos and F. Mugele, Phys. Rev. E: Stat., Nonlinear, Soft Matter Phys., 2010, 81, 015303.

20 H. B. Eral, D. Mampallil and F. Mugele, Europ Patent Office, appl. nr. 10163000.2, 2011.

21 A. R. Wheeler, H. Moon, C. A. Bird, R. R. O. Loo, C. J. Kim, J. A. Loo and R. L. Garrell, Anal. Chem., 2005, 77, 534. 